\title{
Linkage between the bacterial acid stress and stringent responses: the structure of the inducible lysine decarboxylase
}

\author{
Usheer Kanjee ${ }^{1}$, Irina Gutsche ${ }^{2}$, \\ Eftichia Alexopoulos 1,3,6, Boyu Zhao', \\ Majida El Bakkouri ${ }^{1}$, Guillaume Thibault ${ }^{1,7}$, \\ Kaiyin Liu", Shaliny Ramachandran", \\ Jamie Snider ${ }^{1}$, Emil F Pai ${ }^{1,3,4,5}$ and \\ Walid A Houry ${ }^{1, *}$
}

\begin{abstract}
${ }^{1}$ Department of Biochemistry, University of Toronto, Toronto, Ontario, Canada, ${ }^{2}$ Unit for Virus Host-Cell Interactions, Université Joseph Fourier/European Molecular Biology Laboratory Grenoble Outstation/ Centre National de la Recherche Scientifique, Grenoble, France, ${ }^{3}$ Department of Medical Biophysics, University of Toronto, Toronto, Ontario, Canada, ${ }^{4}$ Department of Molecular Genetics, University of Toronto, Toronto, Ontario, Canada and ${ }^{5}$ Division of Cancer Genomics and Proteomics, Ontario Cancer Institute/Princess Margaret Hospital, Campbell Family Institute for Cancer Research, Toronto, Ontario, Canada
\end{abstract}

The Escherichia coli inducible lysine decarboxylase, LdcI/ CadA, together with the inner-membrane lysine-cadaverine antiporter, CadB, provide cells with protection against mild acidic conditions ( $\mathrm{pH} \sim 5)$. To gain a better understanding of the molecular processes underlying the acid stress response, the $\mathrm{X}$-ray crystal structure of LdcI was determined. The structure revealed that the protein is an oligomer of five dimers that associate to form a decamer. Surprisingly, LdcI was found to co-crystallize with the stringent response effector molecule ppGpp, also known as the alarmone, with 10 ppGpp molecules in the decamer. ppGpp is known to mediate the stringent response, which occurs in response to nutrient deprivation. The alarmone strongly inhibited LdcI enzymatic activity. This inhibition is important for modulating the consumption of lysine in cells during acid stress under nutrient limiting conditions. Hence, our data provide direct evidence for a link between the bacterial acid stress and stringent responses.

The EMBO Journal (2011) 30, 931-944. doi:10.1038/

emboj.2011.5; Published online 28 January 2011

Subject Categories: signal transduction; cellular metabolism Keywords: acid stress; alarmone; lysine decarboxylase; oligomerisation; stringent response

\footnotetext{
*Corresponding author. Departments of Biochemistry, University of Toronto, Toronto, Ontario, Canada M5S 1A8. Tel.: + 416946 7141; Fax: + 416978 8548; E-mail: walid.houry@utoronto.ca ${ }^{6}$ Present address: BBS Naturwissenschaften Ludwigshafen, Franz-Zang-Straße 3-7, D-67059 Ludwigshafen, Germany ${ }^{7}$ Current address: Temasek Life Sciences Laboratory, University of Singapore, 117604, Singapore
}

Received: 17 June 2010; accepted: 21 December 2010; published online: 28 January 2011

\section{Introduction}

In the early 1940s, Gale and Epps (1942) observed that Escherichia coli induced a number of amino-acid decarboxylases in response to reduced $\mathrm{pH}$ and that the action of these decarboxylases resulted in the increase of $\mathrm{pH}$ of the growth medium. Four acid stress-induced amino-acid decarboxylases are responsible for this phenomenon. They include the paralogous GadA/GadB glutamic acid decarboxylases (CastanieCornet et al, 1999), the inducible arginine decarboxylase AdiA (Iyer et al, 2003), the inducible ornithine decarboxylase SpeF (Kashiwagi et al, 1991), and the inducible lysine decarboxylase LdcI (also called CadA) (Meng and Bennett, 1992; Snider et al, 2006). Each of these systems has a different $\mathrm{pH}$ optimum and as such is capable of providing protection against acid stress over a wide range of $\mathrm{pH}$ values: down to $\mathrm{pH} 2.0$ for extreme acid stress with GadA/B and AdiA (and to a lesser extent LdcI) and to $\mathrm{pH} 5.0$ with LdcI and SpeF. The enzymes utilize pyridoxal-5'-phosphate (PLP) as a co-factor to decarboxylate the $\alpha$-carbonyl group of their target amino acids in a reaction that consumes an intracellular proton (Foster, 2004). The products of decarboxylation are exchanged for fresh substrate via specific inner-membrane antiporters.

The cytoplasmic inducible lysine decarboxylase (LdcI/ CadA-SwissProt \# P0A9H3) is found together with an innermembrane lysine-cadaverine antiporter CadB (Soksawatmaekhin et al, 2004) on the cadBA operon. Transcription from this operon is controlled by the upstream membrane-bound transcription activator CadC (Watson et al, 1992). Maximum cadBA expression occurs under conditions of low $\mathrm{pH}$ with excess lysine and anaerobiosis. We recently showed that LdcI interacts with a novel MoxR AAA ${ }^{+}$(ATPases associated with various cellular activities) protein named RavA (Snider et al, 2006).

LdcI catalyses the proton-dependent decarboxylation of L-lysine to produce the polyamine cadaverine and carbon dioxide (Sabo et al, 1974). Carbon dioxide diffuses passively out of the cell and the basic cadaverine is excreted into the medium in exchange for fresh lysine by CadB. The cadBA system provides moderate protection for cells exposed to extreme acid shock (pH 2.0-2.5) (Iyer et al, 2003; Snider et al, 2006), but is important for cells growing under less extreme conditions ( $\sim \mathrm{pH} 5.0$ ) (Watson et al, 1992; Dell et al, 1994). Of note, both the gadABC and cadBA systems contribute to the survival of cells grown under conditions that mimic those found in the small intestine, namely: anaerobic environment, phosphate starvation, and presence of fermentation acids (Moreau, 2007). The cadBA system also provides protection against acid stress for pathogenic enterobacteria such as Salmonella enterica serovar Typhimurium (Park et al, 1996) and Vibrio cholerae (Merrell and Camilli, 1999).

Here, we present the X-ray crystal structure of LdcI and show that LdcI has a specific binding pocket for the stringent 
response regulator/alarmone ppGpp. We demonstrate that the activity of LdcI is inhibited in the presence of ppGpp in vitro and in vivo. The induction of the alarmone occurs under various stress conditions or when nutrients become limiting and this has a profound effect on cell physiology. Such effects include the downregulation of stable RNA expression and upregulation of amino-acid biosynthesis genes through the direct binding of ppGpp to the $\beta$ and $\beta^{\prime}$ subunits of RNA polymerase (RNAP) (Artsimovitch et al, 2004; Potrykus and Cashel, 2008), inhibition of DNA replication (Wang et al, 2007), inhibition of protein synthesis (Svitil et al, 1993), induction of the general stress response sigma factor $\sigma^{\mathrm{S}}$ (Cashel et al, 1996), as well as, numerous pleiotropic effects that optimize the cell for survival under stress and starvation conditions.

The novel interaction that we have observed between ppGpp and LdcI is important for regulating the activity of LdcI and provides a previously unknown link between the acid stress and stringent response systems.

\section{Results}

\section{The overall structure of Ldcl}

The X-ray crystal structure of LdcI was determined to a resolution of $2.0 \AA$ with five monomers in the asymmetric unit (refer to Alexopoulos et al (2008) for crystallographic details and to Table I for refinement statistics). The LdcI monomer can be divided into three domains (Figure 1A): an $\mathrm{N}$-terminal wing domain (residues 1-129), a core domain (residues 130-563), and a C-terminal domain (CTD) (residues 564-715). The core domain is made up of a linker region (residues 130-183) followed by two subdomains: a PLPbinding subdomain (PLP-SD) (residues 184-417) corresponding to the large domain of PLP-fold type I enzymes (Käck et al, 1999), and subdomain 4 (SD4) (residues 418-563) corresponding to the small domain of PLP-fold type I enzymes. The wing domain adopts a flavodoxin-like fold and belongs to the CheY-related SCOP-fold family (Murzin et al, 1995), which is characterized by a five-stranded parallel $\beta$-sheet sandwiched between two sets of amphipathic $\alpha$-helices (Figure 1B). The linker region consists of a short helical bundle $(\alpha 5-\eta 2-\alpha 6-\alpha 7)$ that extends outwards from the PLP-SD and is almost at right angles to the wing domain. The PLP-SD has a seven-stranded $\beta$-sheet core surrounded by three sets of $\alpha$-helices. Following strand $\beta 14$ of the $\beta$-sheet core is a short $\alpha$-helix ( $\alpha 13)$ that contains the conserved lysine residue K367 that forms a covalent bond to the PLP co-factor. SD4 stacks on top of PLP-SD and has a central fourstranded antiparallel $\beta$-sheet core and the outer face has three prominent $\alpha$-helices ( $\alpha 16, \alpha 17$, and $\alpha 18$ ), where $\alpha 16$ and $\alpha 18$ are almost parallel and form part of the oligomerisation interface. The CTD has a predominantly $\alpha$-helical outer surface and an inner surface that has two sets of $\beta$-sheets. The CTD folds over the surface formed by PLP-SD and SD4 and forms part of the entry channel into the active site of the enzyme.

The primary unit of the LdcI structure is the two-fold symmetric dimer (Figure 1B) within which the core domains of each monomer are tightly associated, while the wing domains project outwards from the body of the dimer. The majority of the dimer interface is formed by the linker region and PLP-SD with smaller contributions from the remaining protein domains (Supplementary Figure S1). As a consequence of dimerization, the active site of each monomer is buried and the dimer interface forms a narrow cleft that leads into the active site. Dimerization is also necessary for the completion of the active site of each monomer, a common feature in PLP-dependent fold type I decarboxylases (Käck et al, 1999).

The LdcI decamer is $\sim 183 \AA$ wide and $93 \AA$ high and has a central pore of $25 \AA$ diameter as well as five $9 \AA$ wide side pores (Figure $2 \mathrm{~A}$ ). The decamer is formed by the association

Table I Data collection and final refinement statistics for the LdcI crystal structure

\begin{tabular}{|c|c|c|c|c|c|}
\hline \multicolumn{2}{|c|}{ Data collection $^{\mathrm{a}}$} & \multicolumn{4}{|c|}{ Refinement statistics } \\
\hline X-ray source ${ }^{b}$ & BioCARS14-BM-C & Resolution range $(\AA)$ & & $49.9-2.0$ & \\
\hline Wavelength $(\AA)$ & 0.9002 & Reflections used in refinement & & 430750 & \\
\hline Detector & ADSC Quantum 315 & Reflections in test set for $R_{\text {free }}$ & & 22770 & \\
\hline Temperature (K) & 100 & Final $R_{\text {work }} \mathrm{c}^{\mathrm{c}}$ & & 0.169 & \\
\hline Space group & $\mathrm{C} 2$ & Final $R_{\text {free }}$ & & 0.194 & \\
\hline$a(\AA)$ & 271.9 & & Atoms & Ave. & B-factor $\left(\AA^{2}\right)$ \\
\hline$b(\AA)$ & 182.0 & Macromolecule & 28537 & & 26.6 \\
\hline$c(\AA)$ & 171.0 & Ligand & 436 & & 46.9 \\
\hline$\beta$ (deg) & 125.4 & Solvent & 3292 & & 35.5 \\
\hline Unique reflections & 454474 (61 407) & Total & 32266 & & 27.8 \\
\hline Completeness (\%) & $99.8(99.3)$ & RMSD—bond distance $(\AA)$ & & 0.016 & \\
\hline$R_{\text {int }}(\%)^{\mathrm{d}}$ & $16.10(32.66)$ & RMSD_bond angles (deg) & & 1.633 & \\
\hline $\mathrm{I} / \sigma(\mathrm{I})$ & $12.6(3.8)$ & \multicolumn{4}{|c|}{ Ramachandran plot analysis } \\
\hline \multirow{5}{*}{ Redundancy (-fold) } & $5.4(3.4)$ & Most favoured regions (\%) & & 91.2 & \\
\hline & & Additionally allowed regions (\%) & & 8.5 & \\
\hline & & Generously allowed regions (\%) & & 0.4 & \\
\hline & & Disallowed regions (\%) & & 0.0 & \\
\hline & & Omitted residues & & $712-715$ & \\
\hline
\end{tabular}

Values in parentheses refer to the highest $0.1 \AA$ A resolution shell.

${ }^{a}$ Refer to Alexopoulos et al (2008) for initial data collection statistics.

${ }^{b}$ X-ray source refers to the beamline at the Advanced Photon Source (APS), Argonne National Laboratories (ANL), Chicago, IL.

${ }^{\mathrm{c}} R_{\text {work }}=\Sigma\left|F_{\mathrm{o}}-F_{\mathrm{c}}\right| / \Sigma\left|F_{\mathrm{o}}\right|$, where $F_{\mathrm{o}}$ are the observed structure factor magnitudes and $F_{\mathrm{c}}$ are the calculated structure factor magnitudes (Murshudov et al, 1997).

${ }^{\mathrm{d}} R_{\text {int }}=\left[\left(\Sigma_{h} \Sigma_{i}\left|F_{\mathrm{o}}^{2}-<F_{\mathrm{o}}^{2}>\right|\right) /\left(\Sigma_{h} \Sigma_{i}\left|F_{\mathrm{o}}^{2}\right|\right)\right] \times 100$ (Bruker, 2000). 
A

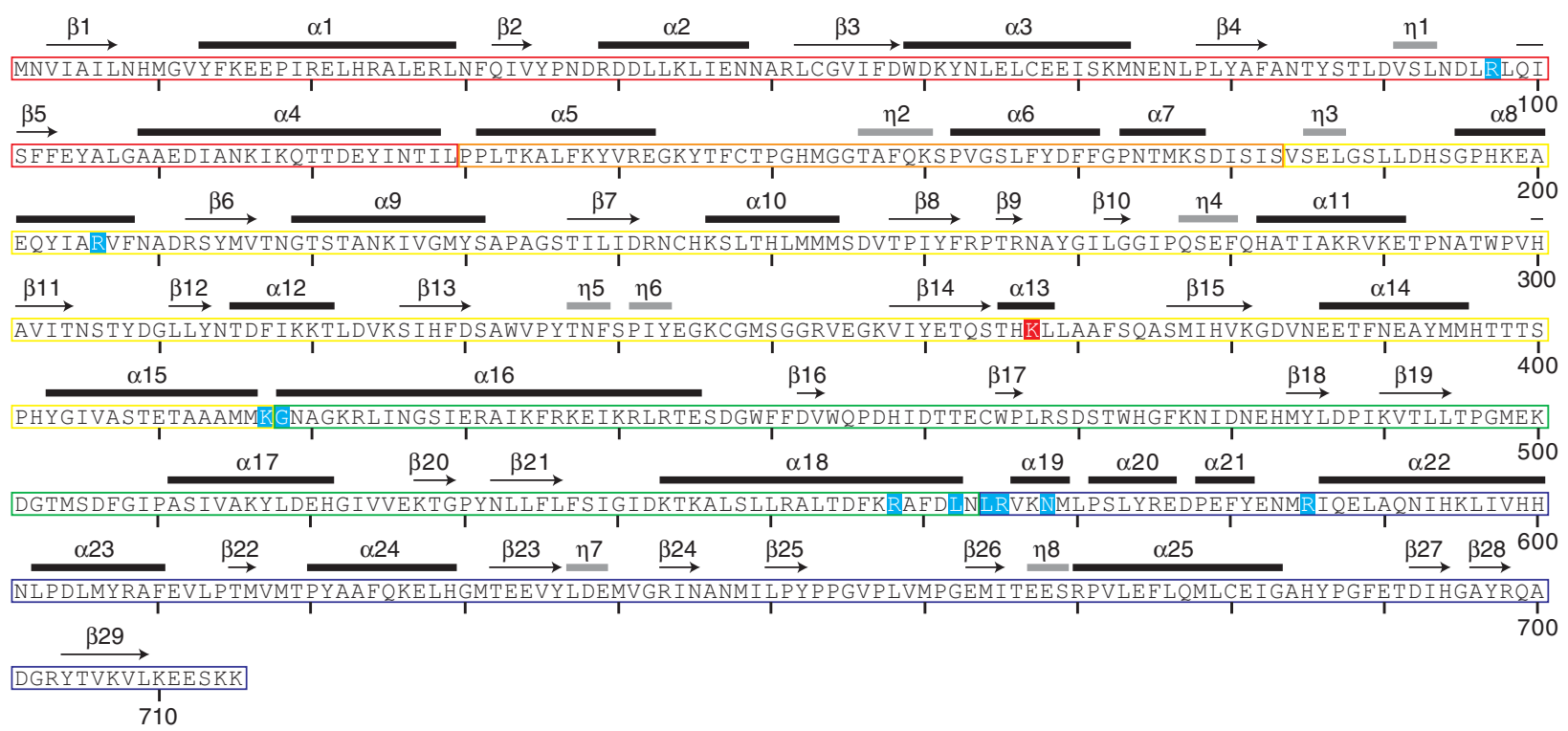

B
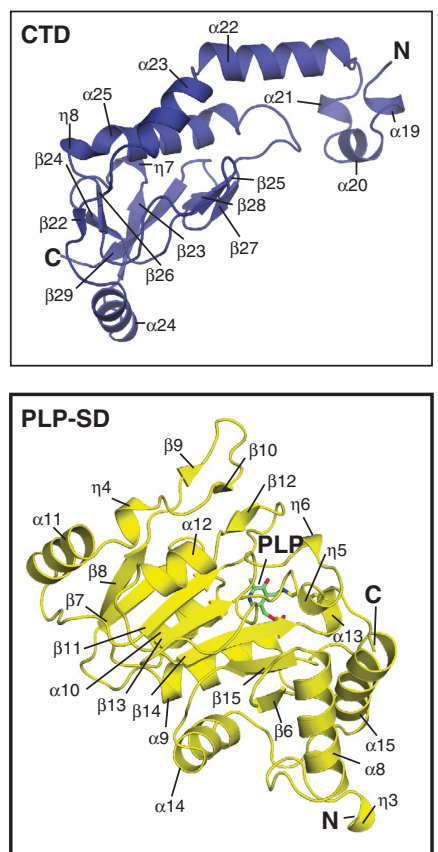

$105 \AA$

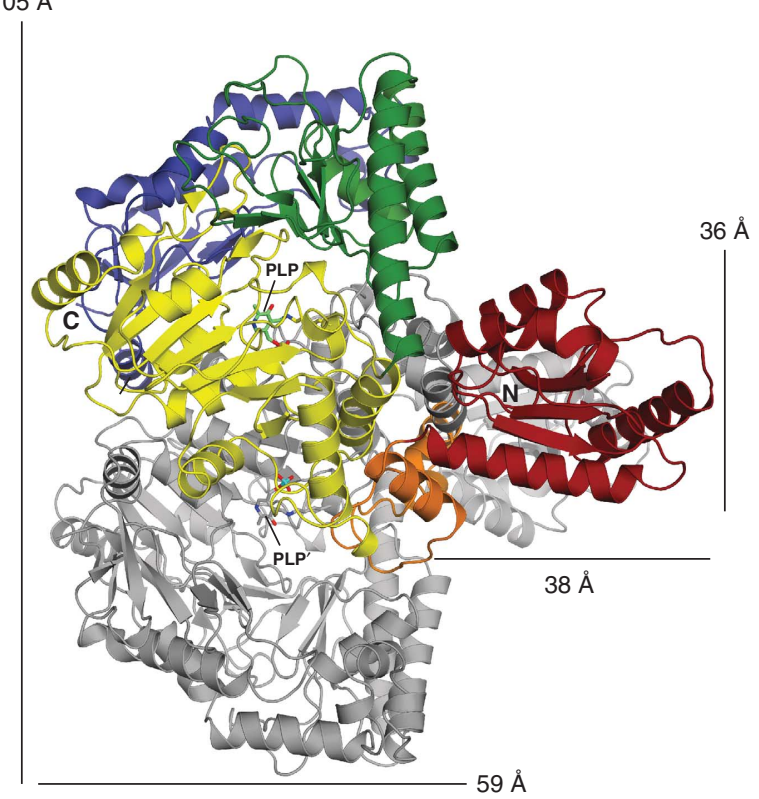

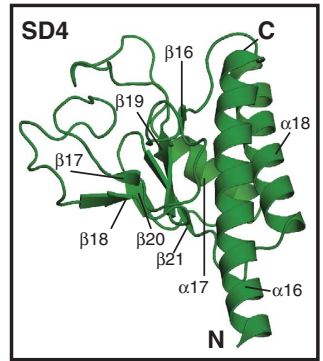
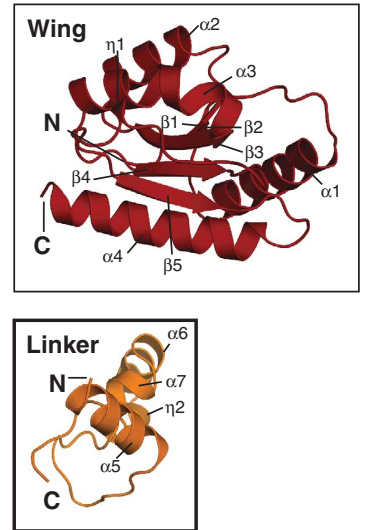

Figure 1 Primary sequence and structure of LdcI dimer. (A) The primary sequence of LdcI is shown with the secondary structure elements indicated above the sequence. The boundaries of the domains are indicated with boxes as follows: N-terminal wing domain-red, linker region-orange, PLP-SD-yellow, SD4-green; and CTD-blue. The conserved lysine (K367) that covalently binds the PLP co-factor is highlighted in red and residues that are involved in binding to ppGpp are highlighted in blue. (B) Cartoon representation of the LdcI dimer is shown orthogonal to the molecular two-fold axis with the domains of the top monomer coloured as in (A) and the bottom monomer coloured grey. Inserts show the secondary structure elements of each of the domains. The subdomains that are part of the core domain are indicated with bold boxes. The PLP co-factor is shown as sticks with the co-factor for the top monomer coloured lime green and the one for the bottom monomer $\left(\mathrm{PLP}^{\prime}\right)$ coloured grey. For PLP, oxygen atoms are in red, nitrogen atoms are in blue, and the phosphate atoms are in cyan. Residues 712-715 were not visible in the electron density map and are not modelled.

of five dimers to form a double-ringed structure with distinct five-fold symmetry as we and others previously observed by negative-staining electron microscopy (EM) (Sabo et al, 1974; Snider et al, 2006) and during the initial determination of the X-ray crystal structure of LdcI (Alexopoulos et al, 2008). In the current work, we obtained an $\sim 20 \AA$ cryoEM reconstruction of LdcI (Figure 2B) that also clearly shows the central and side pores in the decameric structure. A rigid body fit of the X-ray crystal structure, which was determined at $\mathrm{pH} 8.5$, into the cryoEM reconstruction obtained at $\mathrm{pH} 8.0$ (Supplementary Figure S2) indicates that the docking was of high quality, suggesting that the oligomeric conformation is stable and that the obtained X-ray structure of LdcI matches the solution structure of the protein.

Within the context of the LdcI decamer, each monomer participates in two types of interactions: inter-ring/dimer 
A

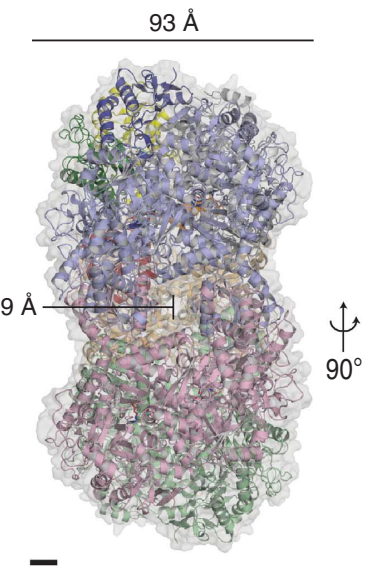

B

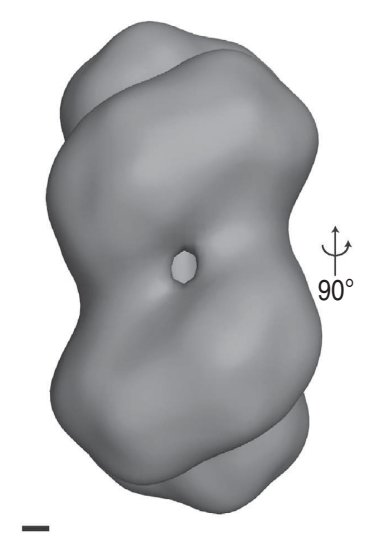

E
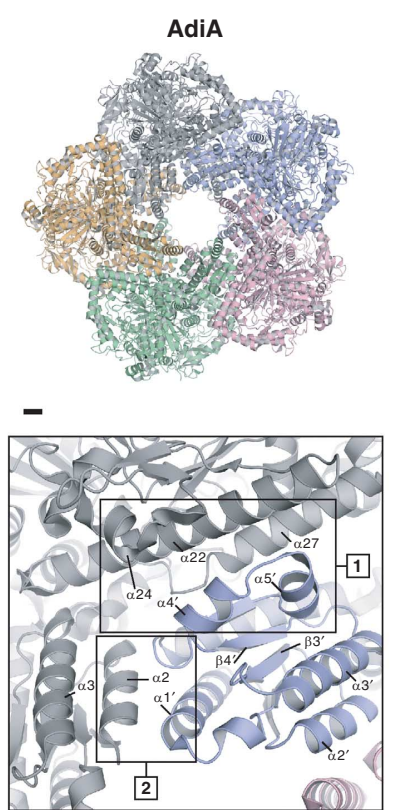

$183 \AA$
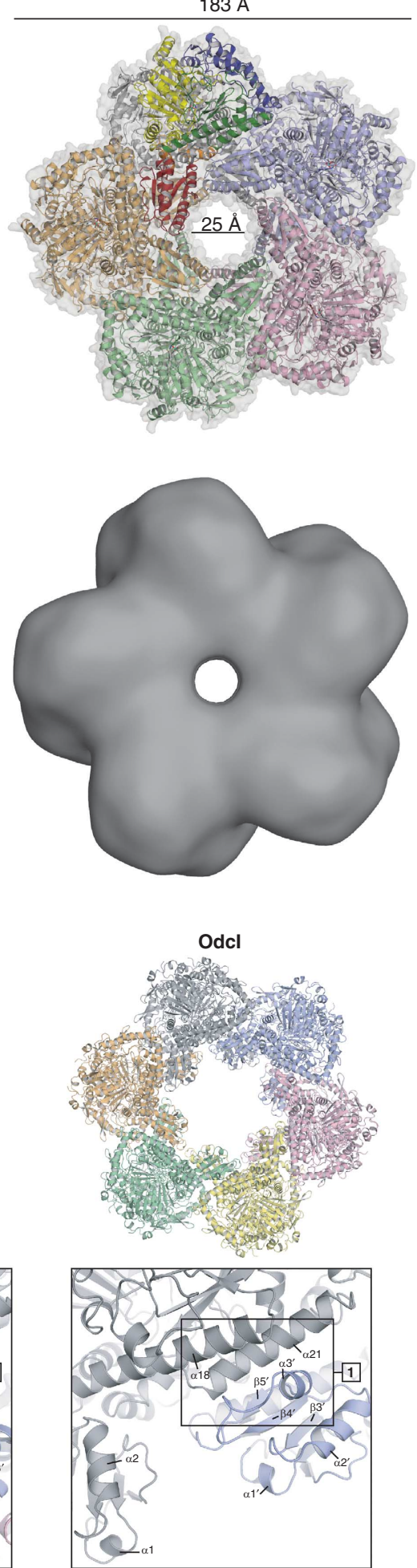

C

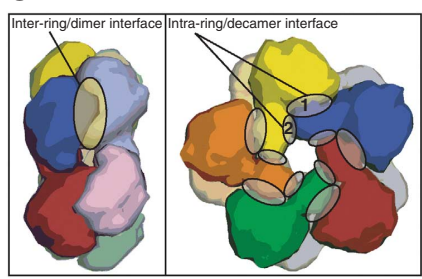

D

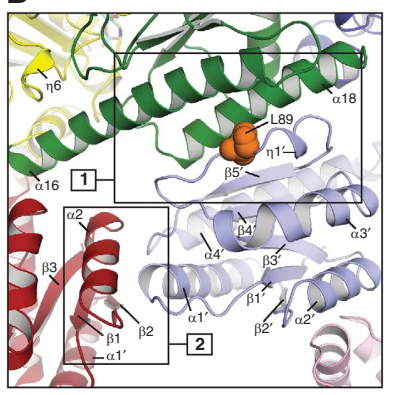

Figure 2 Structure of the LdcI decamer. (A) A cartoon representation of the LdcI decamer is shown beneath a transparent van der Waals' surface. Neighbouring monomers in a dimer are colour matched except for those of the top-most pair that are coloured as in Figure 1B. The dimensions of one of the side pores and the central pore are indicated. All scale bars are $10 \mathrm{~A}$. (B) Side and top views of the cryoEM reconstruction of the LdcI decamer to a resolution of $\sim 20 \AA$ are shown. (C) The inter-ring/dimer interface and the intra-ring/decamer interface are shown schematically. (D) A close-up view of the two interaction interfaces that are responsible for the association of neighbouring dimers into the decamer. Secondary structure elements from the light blue monomer are indicated with a prime symbol. Box 1 and Box 2 interaction interfaces are shown. Residue L89 that is mutated to generate an LdcI dimer (see Figure 6) is labelled. (E) Oligomerisation of AdiA decamer (PDB ID 2VYC) and OdcI dodecamer (PDB ID 1ORD). Dimers in each oligomer are colour matched. Lower panels show close-up views of the oligomerisation interfaces. The wing domain of one grey coloured monomer is shown interacting with the neighbouring monomer in blue. The Box 1 and Box 2 interfaces are shown. 
interactions and intra-ring/decamer interactions (Figure 2C). The intra-ring interactions are between surfaces of the wing and SD4 domains of neighbouring monomers and are indicated as Box 1 and Box 2 in Figure 2D. In Box 1, the interface is formed by the extended loop from $\beta 4^{\prime}$ to $\eta 1^{\prime}$ and parts of $\beta 5^{\prime}$ of the wing domain of one subunit (light blue in Figure 2D) and helices $\alpha 16$ and $\alpha 18$ of SD4 of the neighbouring subunit (coloured green). In Box 2, the interface is formed by residues in $\beta 2$ and $\alpha 2$ in the wing domain of one subunit (coloured red) that interact with the $\mathrm{N}$-terminus of $\alpha 1^{\prime}$ and the loop between $\beta 5^{\prime}$ and the $\mathrm{N}$-terminus of $\alpha 4^{\prime}$ in the wing domain of the neighbouring subunit.

The X-ray crystal structures for two other LdcI homologs have also been determined: the $E$. coli inducible arginine decarboxylase AdiA (PDB ID: 2VYC) (Andrell et al, 2009) and the Lactobacillus 30A ornithine decarboxylase OdcI (PDB ID: 1ORD) (Momany et al, 1995). Structurally, the core and CTDs of the three proteins are very similar. For these domains, the root mean squared deviation between LdcI and AdiA is $1.4 \AA$ for 561 equivalent $C \alpha$ atoms and between LdcI and OdcI is $1.6 \AA$ for 558 equivalent $\mathrm{C} \alpha$ atoms. AdiA oligomerises to form decamers composed of five dimers while OdcI forms a dodecamer composed of six dimers (Figure 2E). All of the structures have the Box 1 interface in common while only the decameric proteins LdcI and AdiA possess the Box 2 interface. This might explain the higher oligomerisation state of OdcI. The Box 1 interface is analogous to the interaction interface formed between $\mathrm{CheY}$, to which the wing domain is structurally related, and its interacting partners CheA, CheZ, and FliM (McEvoy et al, 1999) suggesting that the proteinprotein interaction function of members of the CheY-like fold is conserved.

\section{Ldcl active site}

Members of the fold type I family of PLP-dependent enzymes, despite their wide range of substrate specificities and divergent evolution, maintain a core set of residues and protein contacts that are critical for co-factor binding (Käck et al, 1999; Paiardini et al, 2004). The active site of LdcI is shown in Figure $3 \mathrm{~A}$ and $\mathrm{B}$ and as a schematic in Supplementary Figure S3A. The PLP co-factor was clearly visible in the electron density and is covalently bonded to the $\varepsilon$-amino group of K367 via a Schiff base to form the internal aldimine. During catalysis, the internal aldimine is converted to a lysine-bound external aldimine via a transimination reaction (Christen and Mehta, 2001). The external aldimine tautomerizes to form the quinonoid intermediate that can undergo three possible reactions via loss of one of the substituents at the $\mathrm{C}_{\alpha}$ position. Selectivity of the reaction product is determined by the orientation of the $\pi$-orbitals of the $C_{\alpha}$ substituents as predicted by the Dunathan stereoelectronic hypothesis (Dunathan, 1966; Eliot and Kirsch, 2004).

PLP participates in five sets of interactions with residues in the active site. (1) The phosphate group of PLP forms hydrogen bonds both with residues from the monomer to which the PLP is covalently bound (T220, S221, S364, and H366) as well as to residues from the second monomer in the dimer (T398', $\mathrm{T} 399^{\prime}$, and $\mathrm{S} 400^{\prime}$ ). (2) The electron withdrawing ability of the pyridoxal ring is enhanced (John, 1995) by the structurally conserved salt bridge/hydrogen bond between the pyridine nitrogen N1 and the carboxylate group of D330. (3) The pyridoxal $\mathrm{O} 3$ oxygen atom interacts with the ring nitrogen
NE1 of W333. (4) The imidazole ring of H245 stacks parallel to the re face of the pyridoxal ring. The parallel stacking of an aromatic side chain $(\mathrm{H}, \mathrm{Y}, \mathrm{W}, \mathrm{F})$ in this position favours the formation of the quinonoid intermediate during catalysis (John, 1995; Käck et al, 1999). (5) The methyl group of A332 forms a $\sigma-\pi$ stacking interaction with the si face of the PLP ring consistent with the general stabilization of the si face of the ring with small amino acids (I, V, A, T, S) (Käck et al, 1999). An overlay of the active site of LdcI on that of AdiA or OdcI (Supplementary Figure S3B and C) shows that the residues coordinating PLP are highly conserved.

Analysis of the channels leading to the active sites in LdcI, AdiA, and OdcI dimers showed that LdcI had a larger channel volume compared with that of AdiA and OdcI (Supplementary Figure S4A and B). The residues involved in coordinating the substrate during decarboxylation have not been clearly identified in these enzymes. However, we observed a number of negatively charged residues that line the entry channel to the active site that may interact with the positively charged substrates (Supplementary Figure S4C and D). Residues on the lower part of the cleft are mostly from the SD4 and CT domains of one monomer while residues on the upper part of the cleft are exclusively from the PLP-SD of the opposing monomer. Momany et al (1995) proposed that E532 may be involved in coordinating the ornithine aldimine in OdcI, but this residue, while present in LdcI as E526, is absent in AdiA. In contrast, Andrell et al (2009) proposed that D202' and E739 in AdiA may be important in coordinating the arginine aldimine. Negatively charged residues in equivalent positions straddling the active site cleft are present in LdcI (D192' and D692) and OdcI (D167' and E697). In all cases, interaction with the substrate aldimine might require closure of the active site channel to bring them into close proximity. This effect has been observed most notably in E. coli aspartate aminotransferase where the residue R292 undergoes large movements upon substrate binding leading to formation of the closed complex (Kirsch et al, 1984; Ishijima et al, 2000).

\section{Ldcl binds ppGpp}

During the structure refinement process, we observed that portions of the electron density could not be adequately accounted for either as part of the amino-acid chain of LdcI or by any of the components of the crystallization mixture. After testing different small molecules, we determined that the stringent response effector molecule ppGpp provided the best fit to the electron density with generally good occupancy and reasonable average B-factors (see Supplementary Figure $\mathrm{S} 5 \mathrm{~A}-\mathrm{D}$ for views of the unbiased $2 m F_{\mathrm{o}}-D F_{\mathrm{c}}$ electron density maps). This was an unexpected finding indicating that ppGpp co-purified and then co-crystallized with LdcI. This also suggests that the interaction between LdcI and ppGpp is a specific and strong interaction (see below).

The ppGpp molecules are located at the interface between neighbouring monomers within each ring of the decamer resulting in $10 \mathrm{ppGpp}$-binding sites per decamer (Figure 3A, $\mathrm{C}-\mathrm{F}$ ). The ppGpp-binding pocket is made up of residues from SD4 and CTD of one monomer (coloured light orange in Figure 3C) and the wing domain, PLP-SD, and SD4 of the neighbouring subunit (coloured grey and labelled with a prime symbol). The guanosine base of ppGpp is in the syn conformation and the ribose ring is in the $3^{\prime}$ endo 
conformation. Specificity for the guanosine base is provided for by three residues: the side chain of R558 hydrogen bonds with $\mathrm{O} 6$ and $\mathrm{N} 7$ of the guanosine base; the guanidinium group of R97' stacks against one face of the guanosine base, and the side chain of L564 forms a $\sigma-\pi$ interaction with the opposite face.
The ppGpp-binding pocket has a number of positively charged residues that bind to the phosphate groups of ppGpp. The majority of the interactions are with the $5^{\prime} \alpha$ - and $5^{\prime} \beta$-phosphates, where every oxygen atom except the phospho-esterified oxygen atom participates in an interaction (Figure $3 \mathrm{C}$ and E). The $5^{\prime} \alpha$-phosphate interacts with
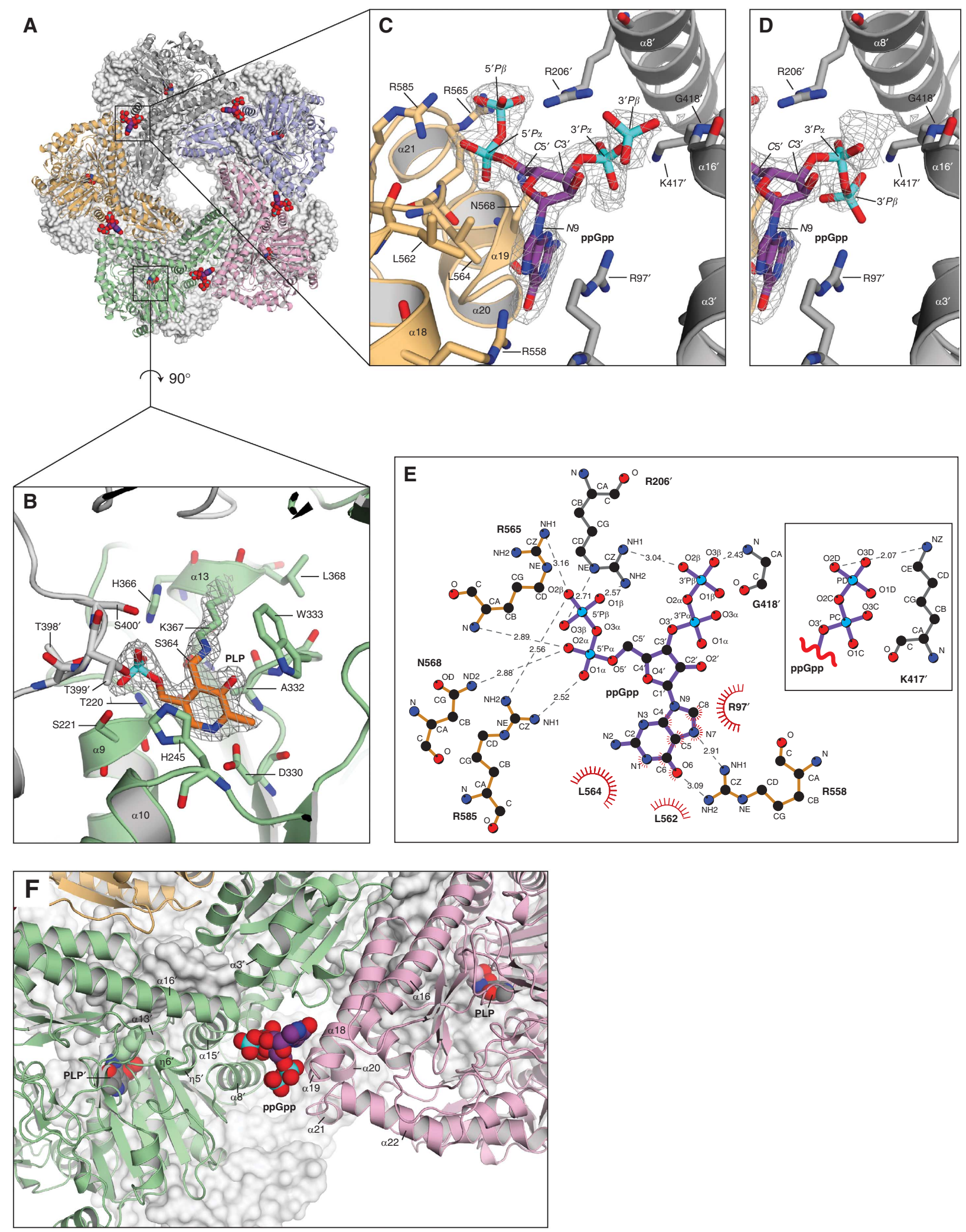
the side chain of R585 and N568, as well as with the backbone amide of R565. The $5^{\prime} \beta$-phosphate interacts with the side chain of R585, the side chain of R565, and the side chain of R206'. Comparison of the residues in equivalent positions to the LdcI ppGpp-binding site in AdiA and OdcI showed that 3 of the 10 residues are strictly conserved (K417, G418, and R206) (Supplementary Figure S5E and F).

In four of the five ppGpp-binding sites within each ring of the LdcI decamer, two conformations for the $3^{\prime} \beta$-phosphate were observed. In the primary conformation of the $3^{\prime} \beta$ phosphate, observed in all five ppGpp-binding sites (Figure $3 \mathrm{C}$ and $\mathrm{E}$ ), the $3^{\prime} \beta$-phosphate forms hydrogen bonds to both the side chain of R206' and the backbone amide of G418'. The secondary conformation (Figure 3D and inset in E) was observed in four of the five binding sites, and the electron density around this phosphate was generally weaker, indicating that this site was occupied less frequently compared with the primary site. Due to the change in position of the $3^{\prime} \beta$ phosphate, the hydrogen bonds with R206' and G418' are lost while a new hydrogen bond is formed with the side chain of K417'. The ppGpp molecule also interacts with a number of water molecules, but none of these interactions are spatially conserved among the five binding sites.

The fact that the ppGpp-binding site was found at an interface between two LdcI protomers and has contributions from residues from several domains led us to speculate that the alarmone might modulate LdcI activity and/or oligomerisation.

\section{Ldcl activity is inhibited by the alarmone}

To determine whether ppGpp has an effect on LdcI activity, we purified apo-LdcI from a $\operatorname{ppGpp}^{0}$ strain and tested the activity of the apoenzyme (at a concentration of $25 \mathrm{nM}$ ) at different $\mathrm{pH}$ values (Figure 4A). In the absence of any nucleotide, LdcI has an activity maximum at $\mathrm{pH} 5.5$ corresponding to an activity of 234 (nmoles cadaverine produced) $\min ^{-1}(\mu \mathrm{g} \mathrm{LdcI})^{-1}$. Addition of ppGpp at low salt concentrations (25-135 mM NaCl depending on the buffer) resulted in a dramatic inhibition of LdcI activity of $\sim 10$-fold at $\mathrm{pH}$ values higher than 5.0 (Figure 4A, upper panel). The pentaphosphate pppGpp also inhibited LdcI but only at $\mathrm{pH}$ values higher than 6.5. The related nucleotides GDP and GTP were ineffective at inhibiting LdcI, indicating that there is a high degree of specificity for (p)ppGpp. At $\mathrm{pH}$ values lower than 5.0 , there is no effect of any nucleotide on LdcI activity.
For free ppGpp, the physiological pKa values of the $3^{\prime} \beta$ and $5^{\prime} \beta$-phosphates were calculated using MARVIN (ChemAxon, 2009) to be 6.52 and 6.82, respectively. Hence, as the $\mathrm{pH}$ increases above these $\mathrm{pKa}$ values, the overall charge of ppGpp becomes more negative resulting in a tighter interaction with LdcI. In addition, the effective range of inhibition was lowered when higher concentrations of either $\mathrm{NaCl}(\geqslant 300 \mathrm{mM})$ (Figure 4A, lower panel) or $\mathrm{KCl}$ $(\geqslant 300 \mathrm{mM})$ (Supplementary Figure S6) were used in the assay, indicating that the binding of ppGpp to LdcI has a strong ionic character. Moreover, the identity of the cation $\left(\mathrm{Na}^{+}\right.$or $\left.\mathrm{K}^{+}\right)$did not affect the activity of LdcI or the inhibition by ppGpp (Supplementary Figure S6).

In order to determine the stoichiometry of binding of ppGpp to LdcI, we performed isothermal titration calorimetry (ITC) at pH 6.5 (Figure 4B). For each LdcI decamer, we observed a total of 10 binding sites with 5 high affinity sites $\left(K_{\mathrm{d}} \sim 13 \mathrm{nM}\right)$ and 5 lower affinity binding sites $\left(K_{\mathrm{d}} \sim 685 \mathrm{nM}\right)$. To explore the mechanism of inhibition, we used an ITCbased enzyme assay to follow the effect of ppGpp on LdcI activity over a range of lysine concentrations (Figure 4C). Apo-LdcI exhibits typical Michaelis-Menten kinetics with a $K_{\mathrm{M}}$ of $0.42 \mathrm{mM}, k_{\text {cat }}$ of $1.80 \times 10^{3} \mathrm{~min}^{-1}$, and a $k_{\text {cat }} / K_{\mathrm{M}}$ of $4.26 \times 10^{6} \mathrm{M}^{-1} \mathrm{~min}^{-1}$. In the presence of ppGpp, the kinetics (Figure 4C) can be modelled to simple inhibition mechanisms that generate comparable kinetic parameters to apo-LdcI with $K_{\mathrm{i}}$ values in the nM to $\mu \mathrm{M}$ range (Figure $4 \mathrm{C}$ ). This suggests that LdcI will be effectively inhibited in vivo at the physiological concentrations of ppGpp (in the $\mathrm{mM}$ range, also see below) reached during the stringent response (Cashel et al, 1996).

\section{Ldcl oligomerisation is modulated by $\mathrm{pH}$ and concentration}

We utilized a number of techniques to probe the oligomerisation of LdcI as a function of $\mathrm{pH}$ and protein concentration. LdcI at $1 \mu \mathrm{M}$ concentration was run on a size exclusion chromatography (SEC) column at different $\mathrm{pH}$ values. At $\mathrm{pH} 5.0$, large aggregates that elute in the void volume of the column were observed (exclusion limit of the column is $\sim 40000 \mathrm{kDa}$ ) while at pH 6.5 and 8.0 decamers were dominant (Figure 5A). Sedimentation velocity analytical ultracentrifugation experiments of $25 \mu \mathrm{M}$ LdcI at $\mathrm{pH} 8.0$ yielded similar results, while experiments at $\mathrm{pH} 6.5$ showed a mixture of LdcI decamers and larger species (Supplementary

Figure 3 PLP and ppGpp-binding sites in LdcI. (A) The top ring of an LdcI decamer is shown as a cartoon representation, where each monomer has a different colour and the bottom ring is shown in grey as a van der Waals' surface. The PLP in each active site and the ppGpp molecules are shown as van der Waals' spheres. Oxygen atoms are in red, nitrogen atoms are in blue, and phosphate atoms are in cyan. (B) The active site of one of the LdcI monomers is shown. Residues that directly interact with PLP are labelled and residues from the second monomer in the dimer that are involved in coordinating the PLP phosphate are shown in grey and labelled with a prime symbol. The grey wire-mesh in parts $\mathrm{B}, \mathrm{C}$, and $\mathrm{D}$ represent the refined $2 m F_{\mathrm{o}}-D F_{\mathrm{c}}$ (Murshudov et al, 1997) electron density map contoured at the $1 \sigma$ level. (C) Shown is a closeup view of one of the ppGpp-binding sites where the left-hand monomer is coloured orange and the right-hand monomer is coloured grey (labels for this monomer are indicated with a prime symbol). The position of the $\mathrm{C}^{\prime}{ }^{\prime}$ and $\mathrm{C} 3^{\prime}$ carbon atoms on the ppGpp ribose ring as well as the phosphate residues are indicated in italics. (D) An alternative conformation of the $3^{\prime} \beta$-phosphate was observed in four of the five ppGpp molecules within each ring of the LdcI decamer and the fit of this conformer to the electron density is shown. (E) Schematic of ppGpp-binding site generated using HBPLUS (McDonald and Thornton, 1994) and LIGPLOT (Wallace et al, 1995). Carbon atoms are shown as black spheres, oxygen atoms are shown as red spheres, nitrogen atoms are shown as blue spheres, and the phosphorous atoms are shown as cyan spheres. ppGpp is shown with purple bonds, residues from one monomer are shown with orange bonds, and residues from the neighbouring monomer are shown with grey bonds. The inset shows the lower occupancy alternative conformation that was observed for the $3^{\prime} \mathrm{P} \beta$ in four of the five ppGpp molecules in the LdcI pentamer. (F) Location of the ppGpp-binding site relative to the neighbouring PLP-binding sites in LdcI is shown. Binding of ppGpp may result in conformational changes that are communicated to the active site of the left-hand monomer (green), potentially via helices $\alpha 8^{\prime}, \alpha 15^{\prime}$, and $\alpha 16^{\prime}$ to helix $\alpha 13^{\prime}$ that bears PLP'. 
A

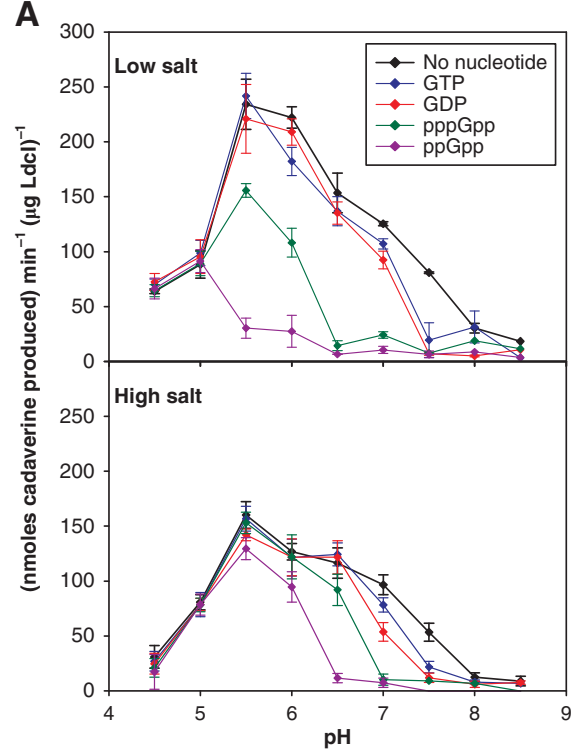

C

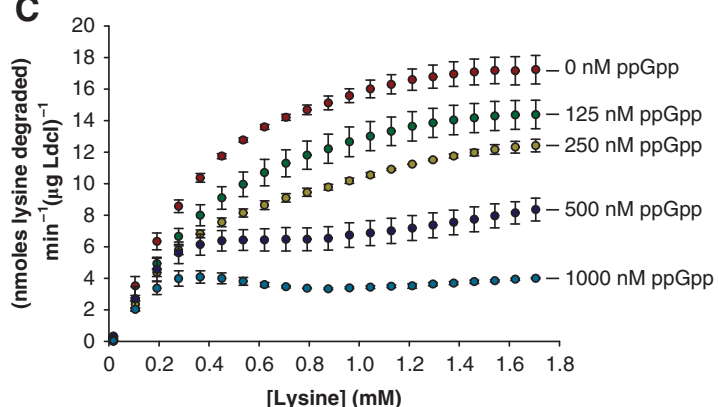

B

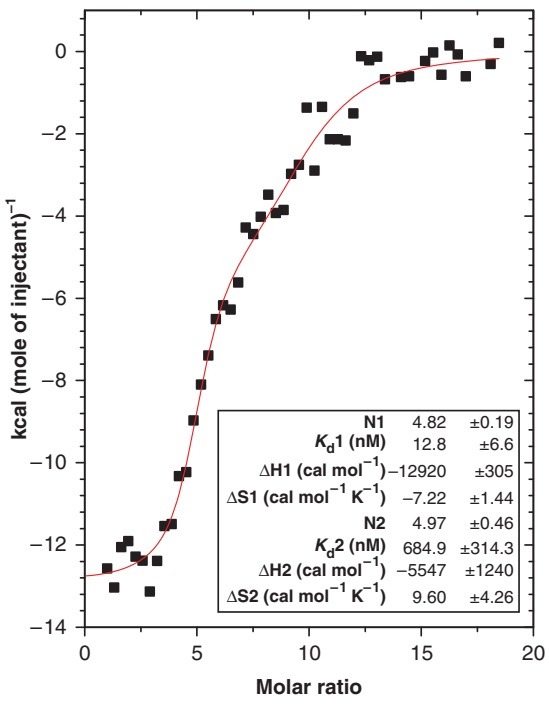

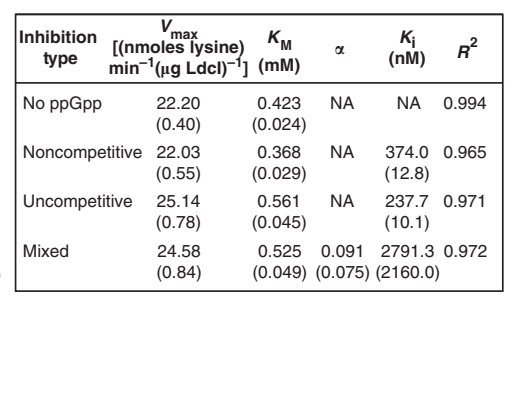

Figure 4 Inhibition of LdcI activity by (p)ppGpp. (A) LdcI $(25 \mathrm{nM})$ activity was measured from pH 4.5 to 8.5 in the absence or presence of $100 \mu \mathrm{M}$ of nucleotides under low salt $(25-135 \mathrm{mM} \mathrm{NaCl}$, upper panel) or high salt ( $\geqslant 300 \mathrm{mM} \mathrm{NaCl}$, lower panel) conditions. Error bars represent the s.d. for the measurements of three repeats. (B) Isothermal titration calorimetry was used to measure ppGpp binding to LdcI at $\mathrm{pH}$ 6.5. Data were fit to a binding model with two non-identical independent binding sites. The inset shows the derived number of binding sites, $K_{\mathrm{d}}, \Delta \mathrm{H}$, and $\Delta \mathrm{S}$ for each type of binding site. (C) Kinetics of LdcI inhibition by ppGpp were followed at pH 6.5 using an isothermal titration calorimetry protocol. Error bars represent the s.d. for the measurements of three repeats. The inset shows the results of the fit of the data. The values in parentheses indicate the standard error from the calculated fits and NA indicates not applicable.

Figure S7A). Negative-staining EM on $\sim 400 \mathrm{nM}$ LdcI (Figure 5B, top row) shows that, at $\mathrm{pH} 5.0$, decamers and higher-order oligomers are present while, at pH 6.5 and 8.0, decamers and an increasing proportion of dimers are present. If we assume equal binding of decamers and dimers to the grid, based on the analysis of 35000 particles, we estimate that at pH 6.5 there are $\sim 25 \%$ dimers and $75 \%$ decamers while at $\mathrm{pH} 8.0$ the proportion of dimers increases to $95 \%$. Glutaraldehyde cross-linking of $25 \mathrm{nM}$ LdcI at various $\mathrm{pH}$ values (Supplementary Figure S7B) shows a similar pattern of large oligomers at $\mathrm{pH} 5.0$ and a mixture of dimers and decamers at higher $\mathrm{pH}$ values.

As the cellular concentration of LdcI increases to about $20 \mu \mathrm{M}$ upon mild acid stress (see below), we additionally tested the oligomerisation and activity at this high-protein concentration. Figure 5C shows that indeed significant inhibition of $20 \mu \mathrm{M}$ LdcI is observed at $\mathrm{pH} 6.5$ and 8.0 but not at $\mathrm{pH}$ 5.0, consistent with the results from enzyme assays conducted at lower protein concentrations (Figure 4A). The SEC profile of $20 \mu \mathrm{M}$ LdcI (Figure 5D) shows that at $\mathrm{pH} 5.0$, a range of large oligomeric species are present and that addition of $100 \mu \mathrm{M}$ ppGpp shifts the distribution towards more decamers, consistent with the EM observations at lower enzyme concentrations (Figure 5B). The addition of ppGpp had no effect on the oligomerisation at either $\mathrm{pH} 6.5$ or $\mathrm{pH}$ 8.0, where a predominance of LdcI decamers was observed. At $20 \mu \mathrm{M}$ LdcI, no significant accumulation of LdcI dimers was visible at any of the $\mathrm{pH}$ values tested. The activities of selected fractions (Figure 5E) from the SEC experiment at $20 \mu \mathrm{M}$ LdcI indicated that at pH 6.5 and 8.0, the decamer is the species that is inhibited by ppGpp. At pH 5.0, the protein activity is distributed over a range of large oligomeric species and these species are not subject to inhibition by ppGpp.

Together, these data suggest that (1) at pH 6.5 and 8.0, LdcI decamer formation is favoured as protein concentration increases and that, at cellular concentrations, LdcI is found predominantly as a decamer. (2) At low protein concentrations, LdcI has a pH-dependent dissociation from decamers to dimers. Class averages of the LdcI dimers in solution show that the overall conformation of the dimers closely matches the conformation that we determined in the crystal structure (Supplementary Figure S7C). This suggests that dimers free in solution maintain a conformation that favours decamer formation. (3) LdcI forms higher-order oligomers at $\mathrm{pH}$ 5.0, the size of which is affected by ppGpp but not their activity. Hence, this suggests that, while ppGpp can bind to LdcI 
A
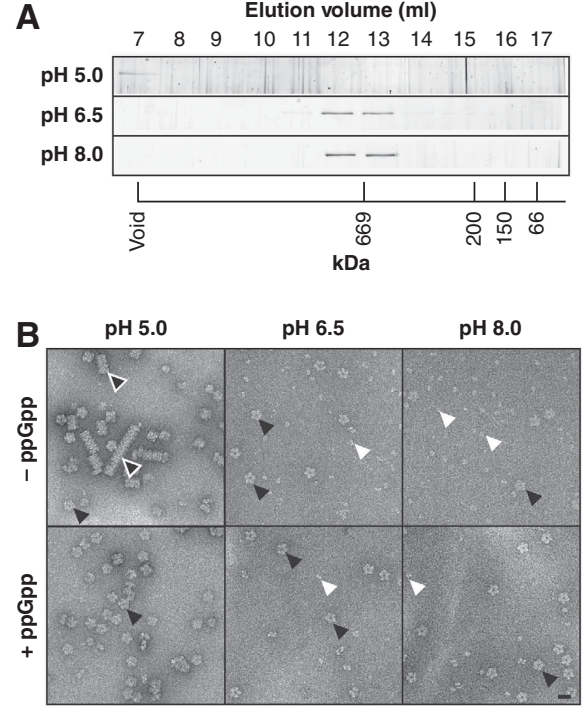

C

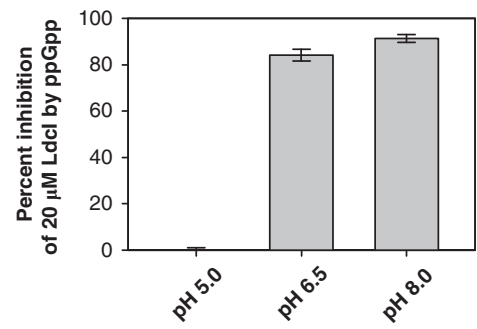

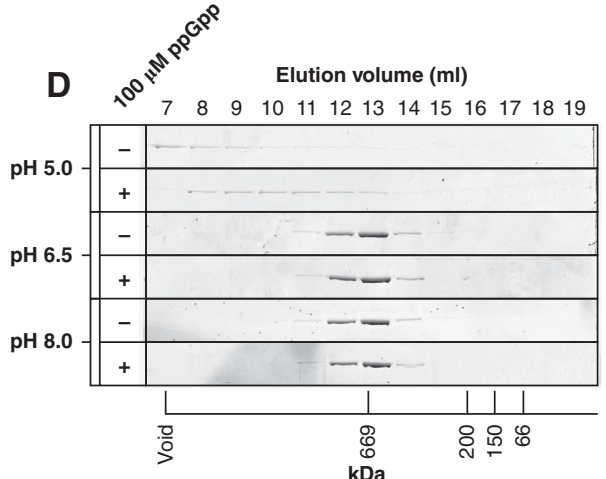

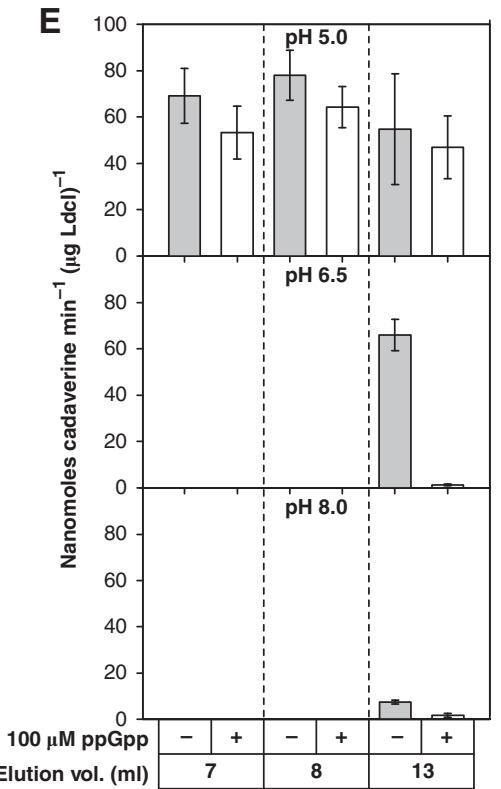

Figure 5 The effect of pH and ppGpp on LdcI oligomerisation. (A) Size exclusion chromatography using a Superose 6 HR 10/30 column of $1 \mu \mathrm{M}$ LdcI at different pHs. The top row indicates $1 \mathrm{ml}$ elution fractions and the positions of molecular weight standards are indicated schematically along the bottom. Each panel represents a slice from a silver-stained polyacrylamide gel. (B) Negative-staining EM of about $400 \mathrm{nM} \mathrm{LdcI}$ at pH 5.0, 6.5, and 8.0 is shown in the absence (top row) and presence (bottom row) of $100 \mu \mathrm{M}$ ppGpp. Decamers are indicated by black arrowheads, dimers are indicated by white arrowheads, and higher-order oligomers are indicated by white bordered black arrowheads. The scale bar is $20 \mathrm{~nm}$. (C) Percent inhibition of activity of $20 \mu \mathrm{M}$ LdcI at pH 5.0, 6.5, and 8.0 by $250 \mu \mathrm{M}$ ppGpp. Error bars represent the s.d. for the measurements of three repeats. (D) Size exclusion chromatography of $20 \mu \mathrm{M} \mathrm{LdcI}$ on a Superose $6 \mathrm{HR} 10 / 30 \mathrm{column}$ at pH 5.0, 6.5, and 8.0 in the absence and presence of $100 \mu \mathrm{M}$ ppGp. Each panel represents a slice from a Coomassie-stained polyacrylamide gel. (E) For selected fractions, the activity of the protein was measured using the TNBS-based LdcI assay either in the absence (grey bars) or presence (white bars) of $100 \mu \mathrm{M}$ ppGpp. Error bars represent the s.d. for the measurements of three repeats.

stacked oligomers at $\mathrm{pH} 5.0$, it is unable to promote the inhibited conformation of the enzyme at this $\mathrm{pH}$. (4) At $\mathrm{pH}$ 6.5 and 8.0 and low protein concentrations, the addition of $100 \mu \mathrm{M}$ ppGpp was seen to increase the proportion of decamers (Figure 5B, bottom row). This apparent stabilization of decamers by ppGpp is consistent with the location of the binding site of ppGpp at the interface between neighbouring monomers in the decamer.

Hence, $\mathrm{pH}$ and concentration have an important role in modulating LdcI oligomerisation and activity.

\section{The effect of alarmone binding to Ldcl on the lysine-dependent $E$. coli acid stress response}

Subsequently, we carried out experiments to determine the significance of ppGpp binding to LdcI on the acid stress response in $E$. coli. To address this question, we first generated two LdcI mutants at the ppGpp-binding interface, R97A and R206S (Figure 3C and D) as well as a mutant that formed a dimer-only version of LdcI, L89R (Figure 2D). The oligomerisation of the different mutants was tested by SEC at $\mathrm{pH}$ 6.5 (Figure 6A). The ppGpp-binding site mutants R97A and R206S showed wild-type oligomerisation profiles while L89R eluted at the expected position for an LdcI dimer (about $150 \mathrm{kDa})$.

The decarboxylation activities of the proteins were then tested at $\mathrm{pH} 5.0$ and 6.5 in the absence and presence of $100 \mu \mathrm{M}$ ppGpp (Figure 6B). At pH 5.0, none of the proteins was inhibited by ppGpp as was observed for WT LdcI. At pH 6.5, WT LdcI was strongly inhibited by ppGpp while the R97A and R206S mutants were insensitive to the addition of ppGpp and had activities comparable to WT LdcI in the absence of ppGpp. The dimeric mutant of LdcI (L89R) showed about five-fold lower level of activity than WT and this activity was not inhibited by ppGpp. A similar trend was 
A
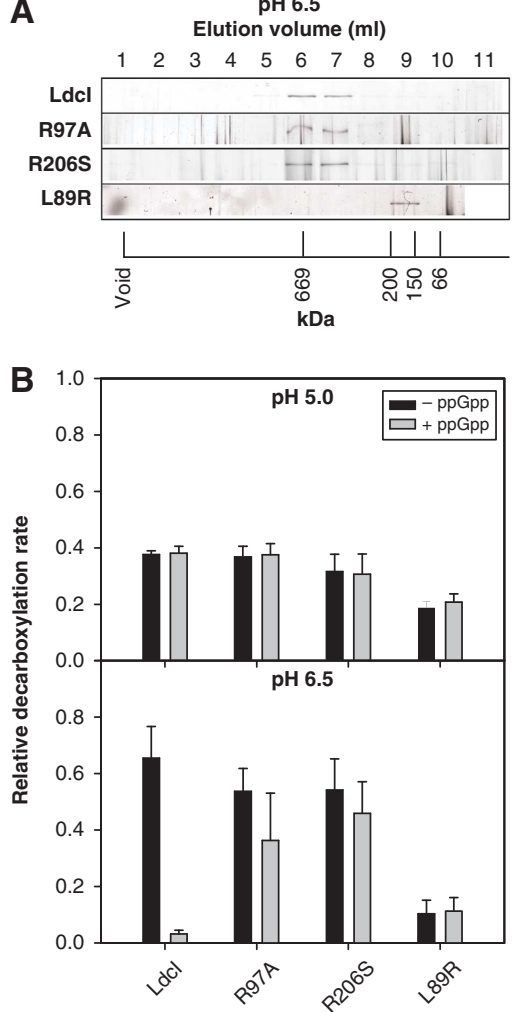

C

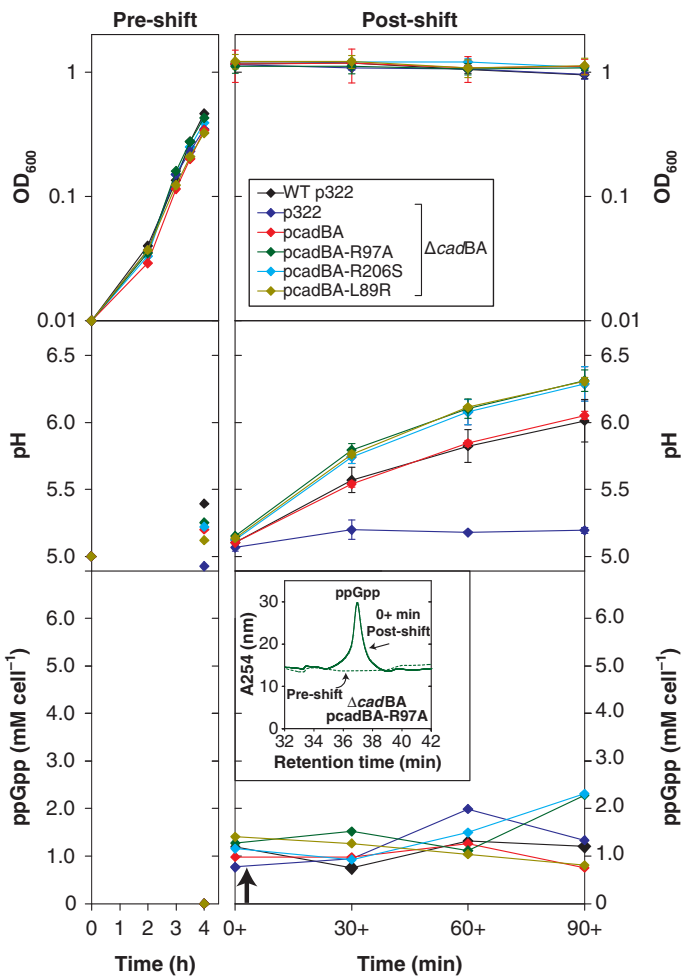

Figure 6 ppGpp modulates the activity of LdcI in the cell. (A) Wild-type and mutant proteins at $1 \mu \mathrm{M}$ were separated on a size exclusion column at pH 6.5 to assess the oligomeric state of LdcI. MW standards are indicated at the bottom. (B) Lysine decarboxylation activity was measured using the TNBS-activity assay in the absence and presence of $100 \mu \mathrm{M}$ ppGpp for WT LdcI and its different mutants at $25 \mathrm{nM}$. Rates are relative to the maximum rate obtained at pH 5.5 (Figure 4A) and error bars indicate the s.d. of the data from three repeats. The top panel shows data at $\mathrm{pH} 5.0$ and the bottom panel shows data at $\mathrm{pH}$ 6.5. (C) Wild-type and $\Delta$ cadBA knockout strains were grown in defined rich media containing $30 \mathrm{mM}$ L-lysine at pH 5.0 until mid-log phase (pre-shift), then the cells were shifted to minimal media also containing $30 \mathrm{mM}$ L-lysine weakly buffered at $\mathrm{pH} 5.0$ (post-shift). The $\mathrm{OD}_{600}$ of the cells is shown in the top panel, the $\mathrm{pH}$ of the culture media is shown in the middle panel, and the amount of ppGpp extracted from the cultures is shown in the bottom panel. The inset shows an HPLC trace demonstrating the induction of ppGpp in the $\Delta$ cadBA pcadBA-R97A strain post-shift. These experiments were repeated three times.

also observed for an additional dimeric mutant (L547R) (Supplementary Figure S8). Hence, LdcI needs to be a decamer in order to bind ppGpp.

To determine the significance of the effect of ppGpp on LdcI activity in vivo, an approach was developed whereby the activity of LdcI in cells was determined by monitoring the change in $\mathrm{pH}$ of weakly buffered media containing L-lysine when cells undergo a stringent response. The following strains were generated and tested: WT MG1655 p322, $\Delta c a d B A$ p322, $\Delta c a d B A$ pcadBA, $\Delta c a d B A$ pcadBA-R97A, $\Delta c a d B A$ pcadBA-R206S, and $\Delta c a d B A$ pcadBA-L89R. p322 is a pET-based plasmid that we constructed (see Supplementary data), while pcadBA refers to the p322 plasmid containing the cadBA operon. The mutations refer to mutations in LdcI.

Cells were grown under LdcI inducing conditions in defined rich media strongly buffered at $\mathrm{pH} 5.0$ and supplemented with all the amino acids plus $30 \mathrm{mM}$ L-lysine. At mid-log phase, the cells were harvested, washed, and re-suspended in minimal media lacking amino acids but supplemented with $30 \mathrm{mM}$ L-lysine to induce a stringent response and weakly buffered at pH 5.0. The activity of LdcI and the levels of ppGpp were then followed as a function of time (Figure 6C). As expected, the $\Delta c a d B A$ strain did not show an increase in the $\mathrm{pH}$ of the media as it lacks the LdcI system but the mutant strains in which LdcI is not inhibited by ppGpp ( $\Delta c a d \mathrm{BA}$
pcadBA-R97A, $\Delta c a d B A$ pcadBA-R206S, and $\Delta c a d B A$ pcadBAL89R) all displayed a more rapid rate of $\mathrm{pH}$ increase than both the WT and $\Delta c a d B A$ pcadBA strains. ppGpp was only detectable post-shift and, within the errors of such measurements, the levels were comparable between the different strains and did not change significantly post-shift (Figure 6C). In addition, the amount of LdcI protein as detected by western blot was generally similar among the different strains (Supplementary Figure S9A and B). To confirm that the difference in the in vivo activity between the LdcI wild type and mutants was dependent on ppGpp, we repeated this shift experiment in a $\Delta r e l \mathrm{~A} \Delta s p o \mathrm{~T} \Delta c a d \mathrm{BA}$ strain complemented with the same plasmids as described above. Deletion of relA and spoT genes renders the strain not capable of generating ppGpp (Xiao et al, 1991). In this case, the wildtype and mutant strains displayed an identical rate of $\mathrm{pH}$ change (Supplementary Figure S10).

It is interesting to note that the in vivo activity of all the LdcI ppGpp-binding site and dimer mutants were similar (Figure 6C; Supplementary Figure S10), even though the LdcI R97A and R206S mutants have about three-fold higher activity than the LdcI dimer mutant L89R when measured in vitro (Figure $6 \mathrm{~B}$ ). This suggests that, under the conditions tested in vivo, such differences cannot be measured or that there are other factors that may have limited the observed 
activity of the proteins such as saturation of the innermembrane lysine-cadaverine antiporter CadB.

The data in Figure 6C indicate that the activity of LdcI in the WT and $\Delta c a d B A$ pcadBA strains was inhibited by the presence of ppGpp when the cells underwent a stringent response. Hence, it could be speculated that, under nutrient limiting conditions, ppGpp is used by the cells to limit LdcI activity upon recovery from acid stress to possibly prevent the over-consumption of lysine.

\section{Discussion}

Our unexpected finding of the modulation of LdcI activity by ppGpp through the direct binding to a specific pocket on the enzyme provides, for the first time, a link between the bacterial acid stress response and the stringent response. It is known that the major effects of the stringent response include the downregulation of stable RNAs, reduction in cellular growth rate, and induction of stress response and amino-acid biosynthesis genes that occur as a result of the change in the transcription profile of RNAP caused by the binding of ppGpp to RNAP (Potrykus and Cashel, 2008). (p)ppGpp is also capable of exerting an effect through direct interaction with targets other than RNAP (Cashel et al, 1996), although, generally, the molecular mechanism by which the alarmone exerts its influence on other proteins is not known.

We found that the LdcI decamers have two types of ppGppbinding sites-low and high affinity sites (Figure 4B). This is surprising from the point of view of the structure as the five ppGpp-binding sites in the asymmetric unit are very similar. However, we do observe two conformations for ppGpp in the binding site, one at higher occupancy and the other at lower occupancy (Figure 3C-E). Binding in the lower occupancy site could be correlated with weaker binding, but other explanations are also possible and might reflect allostery of binding. It should be noted that, when pppGpp was modelled into the binding site, we observed that the $5^{\prime} \gamma$-phosphate was not well supported in the difference map and made few hydrogen bonds with amino acids in the binding site. Combined with the observation that pppGpp inhibits LdcI less efficiently (Figure 4A), this suggests that ppGpp is the preferred inhibitor.

It is not clear at this stage whether other decarboxylases would also be inhibited by the alarmone. However, the fact that many of the related amino-acid decarboxylases (AdiA, SpeF, SpeC, LdcC) in E. coli are known to form oligomers suggests that the inhibition by ppGpp might be a general method for regulating these enzymes, some of which participate in the acid stress response and some of which are critical for production of polyamines. Further experimentation will be needed to establish this link.

In order to determine the effects of ppGpp binding on the LdcI structure, we crystallized apo-LdcI. So far, the diffraction pattern from the best crystal only extends to $4.1 \AA$ (Supplementary Figure S11; Supplementary Table S1). At this resolution, we would be able to observe only largescale conformational changes between LdcI-ppGpp and apo-LdcI or major rearrangements of the active site or ppGpp-binding site. However, no such differences have been seen. Given the uncertainties in the placement of side-chain groups at this resolution, we cannot comment on the possibility that ppGpp binding induces subtle changes that lead to inhibition of LdcI. As binding of the alarmone does cause inhibition, we speculate that upon ppGpp binding, smaller structural changes may be transduced via helices $\alpha 8, \alpha 15$, and $\alpha 16$ to helix $\alpha 13$ that bears PLP (Figure 3F). We are undertaking a systematic mutational and enzymatic analysis coupled with obtaining the structure of both the apoenzyme and holoenzyme at different pHs in order to gain a more detailed understanding of the mechanism of ppGpp inhibition.

In vitro, LdcI is seen to adopt a number of different oligomeric states depending on protein concentration and pH: namely, higher-order stacked oligomers, decamers, and dimers. These diverse oligomeric states of LdcI exist under different conditions, have different activities, and are differently affected by ppGpp. At high-protein concentrations, about tens of $\mu \mathrm{M}$, LdcI forms stacked oligomers at $\mathrm{pH} 5$ or lower and mainly decamers at pH 6.5 or higher, but no significant percentage of dimers are observed at these highenzyme concentrations (Figure 5D; Supplementary Figure S7A). At low LdcI concentrations, $<1 \mu \mathrm{M}$, the enzyme forms stacked oligomers at $\mathrm{pH} 5$ or lower, while, at pH 6.5 or higher, decamers and dimers are observed with the percentage of dimers increasing as $\mathrm{pH}$ increases (Figure 5A and B; Supplementary Figure S7B). Hence, there are multiple mechanisms by which the cell can regulate LdcI activity to either counter acid stress or to facilitate recovery from acid stress.

Under mild acid stress conditions with an external $\mathrm{pH}$ of 4-5, the internal $\mathrm{pH}$ inside the cell is expected to be $6-7$ (Richard and Foster, 2004); under these conditions, LdcI will be highly induced (i.e. will be at high concentrations) and the decameric form of the enzyme will be the major species. If cells reach stationary phase or face starvation, then ppGpp is induced and LdcI decamer activity will be inhibited. Furthermore, as the cells recover from acid stress, LdcI levels will decrease as transcription from the cadBA operon is abolished (Watson et al, 1992) halting LdcI production and the protein concentration will be reduced due to cell division or degradation activity. As LdcI concentration is reduced and the internal $\mathrm{pH}$ is increased, the proportion of dimers is expected to increase as suggested by the experiments of Figure 5B. Based on the measured activity of the two dimer-only mutants, L89R (Figure 6B) and L547R (Supplementary Figure S8B), dimers have lower specific activity than decamers. We expect this lower activity of LdcI dimers to be important for cells recovering from acid stress in the absence of a stringent response. This should eventually result in the reduction of LdcI activity and stop the consumption of lysine by the enzyme. The cellular lysine pool would then be fully available for other functions such as protein synthesis.

Under extreme acid stress conditions with an external $\mathrm{pH}$ of 2-3 (Richard and Foster, 2004) or in the presence of organic acids (Wilks and Slonczewski, 2007), the internal $\mathrm{pH}$ will be $4-5$. LdcI is not expected to be significantly induced under such conditions. However, if LdcI is present under these conditions, whether at low or high concentrations, it is expected to form higher-order oligomers composed of stacks of decamers (Figure 5A, B, and D). At this pH, LdcI is insensitive to ppGpp (Figures $4 \mathrm{~A}$ and $5 \mathrm{E}$ ) and this may be an important adaptation to allow LdcI to function, albeit at a reduced activity, when the internal $\mathrm{pH}$ of the cytoplasm falls 
to below pH 5 (i.e. external pH of about 3 ) during severe acid challenges.

The above scenarios reveal a very interesting dilemma. If cells are faced with acidic stress under nutrient limiting conditions, then high levels of amino-acid decarboxylases such as LdcI are required to raise a proper response; however, at the same time, cells need to conserve the amount of amino acids consumed during the decarboxylation reactions and to quickly shut down such enzymes when the $\mathrm{pH}$ approaches neutral. The use of ppGpp to allosterically inhibit enzymatic decarboxylation activity seems to be a very efficient method to fine-tune the bacterial acid stress response. It should be noted in this regard that, since the weaker $K_{\mathrm{d}}$ for ppGpp binding to LdcI is $685 \mathrm{nM}$, we expected LdcI to be completely inhibited during the stringent response when the concentration of ppGpp is increased to $\sim 1-2 \mathrm{mM}$ (Figure $6 \mathrm{C}$ ), the concentration of LdcI is $\sim 20-30 \mu \mathrm{M}$ (Supplementary Figure S9B) and the cytoplasmic $\mathrm{pH}$ is expected to be close to neutral (Wilks and Slonczewski, 2007). Under these conditions, we expect LdcI to be predominantly decameric and to have its activity regulated primarily by binding ppGpp. Indeed, we do observe lower LdcI activity for the WT protein versus the ppGpp-binding site mutants in vivo (Figure 6C); however, the incomplete inhibition of the protein suggests the possibility of additional systems that might regulate the inhibition of LdcI by ppGpp binding. Hence, we suspect the presence of other systems that might fine-tune the activity of LdcI.

\section{Materials and methods}

\section{Biochemical assays}

The activity of lysine decarboxylase was measured using either a 2,4,6-trinitrobenzensulfonic acid (TNBS) assay as described (Snider et al, 2006; Kanjee and Houry, 2010) or via ITC (using a Microcal VP-ITC) to measure the heat of the reaction of lysine decarboxylation under a range of substrate and inhibitor concentrations. The VP-ITC was used to measure the binding of ppGpp to LdcI following standard procedures.

SEC experiments were performed in $100 \mathrm{mM}$ buffer (sodium acetate for $\mathrm{pH}$ 5.0, sodium MES for $\mathrm{pH}$ 6.5, and sodium HEPES for $\mathrm{pH}$ 8.0), $300 \mathrm{mM} \mathrm{NaCl}, 0.1 \mathrm{mM}$ PLP, and $1 \mathrm{mM}$ DTT and run on either an ÄKTA FPLC (GE) or a Biologic DuoFlow (Bio-Rad) chromatography system and all experiments were performed between 4 and $10^{\circ} \mathrm{C}$. Protein absorbance was determined at $280 \mathrm{~nm}$ and fraction sizes were $1 \mathrm{ml}$ unless otherwise noted. Samples were run on either a Superose 6 HR 10/30 column (GE) or a Superdex 200 10/300 GL column (GE).

\section{In vivo assays}

The effect of binding of ppGpp to LdcI was measured in vivo as follows. Cells were grown to an $\mathrm{OD}_{600}$ of between 0.4 and 0.5 with shaking at $37^{\circ} \mathrm{C}$ in defined rich media (Neidhardt et al, 1974) modified as follows: media were supplemented with $40 \mathrm{mM}$ MES and adjusted to $\mathrm{pH} 5.0$ with $\mathrm{KOH}$; the concentration of all 20 amino acids was $50 \mathrm{mg} \mathrm{ml}^{-1}$; the carbon source was $0.1 \%(\mathrm{w} / \mathrm{v}$ ) D-glucose and the media were supplemented with $30 \mathrm{mM}$ L-lysine. At mid-log phase, cells were pelleted by centrifugation at $4000 \mathrm{~g}$ at $22^{\circ} \mathrm{C}$ for $10 \mathrm{~min}$, washed once in the post-shift media $(5 \mathrm{mM}$ MES pH 5.0, $1.32 \mathrm{mM} \mathrm{K}_{2} \mathrm{HPO}_{4}, 0.2 \mathrm{mM}$ each of adenine, cytosine, guanine, and uracil, and $0.001 \%(\mathrm{w} / \mathrm{v}) \mathrm{D}$-glucose), pelleted again as above and finally re-suspended to an $\mathrm{OD}_{600}$ of 1.0 in post-shift media containing $30 \mathrm{mM}$ L-lysine. The cells were incubated at $37^{\circ} \mathrm{C}$ with shaking for $2 \mathrm{~h}$ and samples were withdrawn every half an hour for determination of the amount of LdcI and ppGpp and measurement of the $\mathrm{OD}_{600}$ and $\mathrm{pH}$. The extraction of ppGpp from the cell culture was performed as described (Buckstein et al, 2008) with minor modifications.

Other details and biochemical protocols are provided in Supplementary data.

\section{Ldcl crystallization and structure determination}

The purification, crystallization, data collection, and preliminary structure determination of LdcI-ppGpp have been described previously (Alexopoulos et al, 2008). A detailed description of the structure determination process is presented in Supplementary data. We obtained initial low-resolution maps at $4.5 \mathrm{~A}$ by single isomorphous replacement with anomalous scattering (SIRAS) using LdcI crystals soaked in the heavy atom cluster hexatantalum dodecabromide $\left(\mathrm{Ta}_{6} \mathrm{Br}_{12}^{2+}\right)$. The initial electron density maps were not of sufficient quality to trace the protein backbone but enabled us to generate a molecular replacement (MR) search model based on the structure of the orthologous Lactobacillus 30A ornithine decarboxylase OdcI (PDB ID: 1ORD) that shares $31.4 \%$ sequence identity with LdcI and has a similar domain architecture. We obtained an MR solution in PHASER (McCoy et al, 2007) consisting of five monomers in a star-like arrangement. The initial refinement was started in REFMAC (Murshudov et al, 1997) using the Native-1 data set (Alexopoulos et al, 2008) to $2.57 \AA$ resolution, and the refinement was extended to $2.0 \AA$ with the current high-resolution data set (Table I). Five percent of the reflections were randomly selected as an $R_{\text {free }}$ set for cross-validation. Initial rigid body refinement was followed by a refinement using tight non-crystallographic symmetry restraints. Model building was performed interactively using COOT (Emsley and Cowtan, 2004). During the final refinement steps, the tight NCS restraints were released. Final refinement statistics are given in Table I. The coordinates have been deposited in the RCSB with PDB ID 3N75. All structure figures were prepared in PyMOL (DeLano, 2002). The structure determination of apo-LdcI is described in Supplementary data with the respective coordinates deposited in the RCSB with PDB ID 3Q16.

\section{Electron microscopy}

CryoEM reconstruction of LdcI was performed on purified protein as follows. The vitrified specimen was prepared on holey carbon copper grids as described (Dubochet et al, 1988) and observed with a Phillips CM200 transmission electron microscope with a $\mathrm{LaB}_{6}$ filament at $200 \mathrm{kV}$. Images were recorded under low electron dose conditions on Kodak SO-163 films and negatives were digitized with a Nikon Coolscan 6000 scanner at $3.34 \AA$ pixel size at the specimen level. The defocus of the images used for further analysis was $\sim 2-$ $2.5 \mu \mathrm{m}$ as determined from the power spectra. Image processing was carried out using the EMAN software package (Ludtke et al, 1999) for particle selection, CTFFIND (Mindell and Grigorieff, 2003) for CTF determination and correction, Imagic-5 (van Heel et al, 1996) for classification and angular reconstitution and EMAN refine procedure as an alternative 3D-reconstruction option. No matter whether our previously described 3D reconstruction based on negative-stain images of the LdcI decamer (Snider et al, 2006) was used as an initial model or whether $a b$ initio calculations were performed, the final reconstruction was the same. For the purpose of this work and because of the notable similarity with the X-ray crystal structure, we filtered this reconstruction to $20 \AA$ resolution and did not attempt to improve it further.

For preparation of negatively stained LdcI, protein solution was applied to the clear side of carbon on a carbon-mica interface and stained with $2 \%(\mathrm{w} / \mathrm{v})$ silicotungstate. The $\mathrm{pH}$ of the stain was adjusted with $\mathrm{NaOH}$ to be $5.0,6.5$, or 8.0 to correspond exactly to the $\mathrm{pH}$ of the sample buffer. Images were recorded under low-dose conditions with a JEOL 1200 EX II microscope at $100 \mathrm{kV}$.

\section{Supplementary data}

Supplementary data are available at The EMBO Journal Online (http://www.embojournal.org).

\section{Acknowledgements}

We thank Dr Michael Cashel (National Institutes of Health, Bethesda, MD) for sending us bacterial strains, plasmids, and necessary protocols. We thank Dr Eric Massé (University of Sherbrooke, Québec City, Canada) for the gift of purified P1 phage. We thank Dr Tyrell Conway (University of Oklahoma, OK) for the gift of the MG1655 $\Delta$ relA $\Delta s p o T$ strain. UK is the recipient of a National Sciences and Engineering Research Council of Canada (NSERC) Postgraduate Scholarship, a Fellowship of the Canadian Institutes of Health Research (CIHR) Strategic Training Program in the Structural Biology of Membrane Proteins Linked to Disease, and 
a University of Toronto Open Fellowship. EA is the recipient of a fellowship from the CIHR Strategic Training Program in Protein Folding: Principles and Diseases. BZ is the recipient of an NSERC Undergraduate Student Research Award (NSERC-USRA). KY is the recipient of a Life Sciences Award from the University of Toronto. MEB is the recipient of a fellowship from the CIHR Strategic Training Program in Protein Folding and Interaction Dynamics: Principles and Diseases. SR is the recipient of an NSERC-USRA and the University of Toronto Excellence Award in Natural Sciences and Engineering. GT and JS are recipients of an NSERC Postgraduate Scholarship. This work was supported by a grant from the CIHR (MOP-67210) to WAH, by the Canada Research Chairs Program and the Ontario Research and Development Challenge Fund to EFP and in part by the Ontario Ministry of Health and Long Term Care. The views expressed do not necessarily reflect those of the OMOHLTC. The results shown in this manuscript are derived from work

\section{References}

Alexopoulos E, Kanjee U, Snider J, Houry WA, Pai EF (2008) Crystallization and preliminary X-ray analysis of the inducible lysine decarboxylase from Escherichia coli. Acta Crystallogr Sect $F$ Struct Biol Cryst Commun 64 (Pt 8): 700-706

Andrell J, Hicks MG, Palmer T, Carpenter EP, Iwata S, Maher MJ (2009) Crystal structure of the acid-induced arginine decarboxylase from Escherichia coli: reversible decamer assembly controls enzyme activity. Biochemistry 48: 3915-3927

Artsimovitch I, Patlan V, Sekine S, Vassylyeva MN, Hosaka T, Ochi K, Yokoyama S, Vassylyev DG (2004) Structural basis for transcription regulation by alarmone ppGpp. Cell 117: 299-310

Bruker (2000) SMART, SAINT, SADABS, and XPREP Software Reference Manual. Madison, WI: Bruker AXS Inc.

Buckstein MH, He J, Rubin H (2008) Characterization of nucleotide pools as a function of physiological state in Escherichia coli. J Bacteriol 190: 718-726

Cashel M, Gentry DR, Hernandez VJ, Vinella D (1996) The stringent response. In Escherichia coli and Salmonella: Cellular and Molecular Biology, Curtiss R, Neidhardt FC (eds), 2nd edn, pp 1458-1496. Washington, D.C.: ASM Press

Castanie-Cornet MP, Penfound TA, Smith D, Elliott JF, Foster JW (1999) Control of acid resistance in Escherichia coli. J Bacteriol 181: 3525-3535

ChemAxon (2009) Marvin, version 5.2. Budapest

Christen P, Mehta PK (2001) From cofactor to enzymes. The molecular evolution of pyridoxal-5'-phosphate-dependent enzymes. Chem Rec 1: 436-447

DeLano WL (2002) The PyMOL Molecular Graphics System. Palo Alto, CA: DeLano Scientific

Dell CL, Neely MN, Olson ER (1994) Altered pH and lysine signalling mutants of cadC, a gene encoding a membrane-bound transcriptional activator of the Escherichia coli cadBA operon. Mol Microbiol 14: 7-16

Dubochet J, Adrian M, Chang JJ, Homo JC, Lepault J, McDowall AW, Schultz P (1988) Cryo-electron microscopy of vitrified specimens. Q Rev Biophys 21: 129-228

Dunathan HC (1966) Conformation and reaction specificity in pyridoxal phosphate enzymes. Proc Natl Acad Sci USA 55: 712-716

Eliot AC, Kirsch JF (2004) Pyridoxal phosphate enzymes: mechanistic, structural, and evolutionary considerations. Annu Rev Biochem 73: 383-415

Emsley P, Cowtan K (2004) Coot: model-building tools for molecular graphics. Acta Crystallogr D Biol Crystallogr 60 (Pt 12 Pt 1): 2126-2132

Foster JW (2004) Escherichia coli acid resistance: tales of an amateur acidophile. Nat Rev Microbiol 2: 898-907

Gale EF, Epps HM (1942) The effect of the $\mathrm{pH}$ of the medium during growth on the enzymic activities of bacteria (Escherichia coli and Micrococcus lysodeikticus) and the biological significance of the changes produced. Biochem J 36: 600-618

Ishijima J, Nakai T, Kawaguchi S, Hirotsu K, Kuramitsu S (2000) Free energy requirement for domain movement of an enzyme. J Biol Chem 275: 18939-18945

Iyer R, Williams C, Miller C (2003) Arginine-agmatine antiporter in extreme acid resistance in Escherichia coli. J Bacteriol 185: 6556-6561 performed at Argonne National Laboratory, beamline 19-BM-D, Structural Biology Center and beamline 14-BM-C, BioCARS sector, both at the Advanced Photon Source. Argonne is operated by UChicago Argonne, LLC, for the US Department of Energy, Office of Biological and Environmental Research under contract DE-AC0206CH11357. The use of the BioCARS Sector 14 was supported by the National Institutes of Health, National Center for Research Resources under grant No. RR0077-7. We also thank the members of Canadian Light Source (CLS) beamline staff at CMCF-08ID-1 for assistance with data collection.

\section{Conflict of interest}

The authors declare that they have no conflict of interest.

John RA (1995) Pyridoxal phosphate-dependent enzymes. Biochim Biophys Acta 1248: 81-96

Käck H, Sandmark J, Gibson K, Schneider G, Lindqvist Y (1999) Crystal structure of diaminopelargonic acid synthase: evolutionary relationships between pyridoxal-5'-phosphate-dependent enzymes. J Mol Biol 291: 857-876

Kanjee U, Houry WA (2010) An assay for measuring the activity of Escherichia coli inducible lysine decarboxylase. J Vis Exp 46: (doi:10.3791/2094; http://www.jove.com/details.stp?id = 2094)

Kashiwagi K, Suzuki T, Suzuki F, Furuchi T, Kobayashi H, Igarashi K (1991) Coexistence of the genes for putrescine transport protein and ornithine decarboxylase at $16 \mathrm{~min}$ on Escherichia coli chromosome. J Biol Chem 266: 20922-20927

Kirsch JF, Eichele G, Ford GC, Vincent MG, Jansonius JN, Gehring $\mathrm{H}$, Christen P (1984) Mechanism of action of aspartate aminotransferase proposed on the basis of its spatial structure. $J \mathrm{Mol}$ Biol 174: 497-525

Ludtke SJ, Baldwin PR, Chiu W (1999) EMAN: semiautomated software for high-resolution single-particle reconstructions. J Struct Biol 128: 82-97

McCoy AJ, Grosse-Kunstleve RW, Adams PD, Winn MD, Storoni LC, Read RJ (2007) Phaser crystallographic software. J Appl Crystallogr 40: 658-674

McDonald IK, Thornton JM (1994) Satisfying hydrogen bonding potential in proteins. J Mol Biol 238: 777-793

McEvoy MM, Bren A, Eisenbach M, Dahlquist FW (1999) Identification of the binding interfaces on CheY for two of its targets, the phosphatase CheZ and the flagellar switch protein FliM. J Mol Biol 289: 1423-1433

Meng SY, Bennett GN (1992) Nucleotide sequence of the Escherichia coli cad operon: a system for neutralization of low extracellular pH. J Bacteriol 174: 2659-2669

Merrell DS, Camilli A (1999) The cadA gene of Vibrio cholerae is induced during infection and plays a role in acid tolerance. Mol Microbiol 34: 836-849

Mindell JA, Grigorieff N (2003) Accurate determination of local defocus and specimen tilt in electron microscopy. J Struct Biol 142: 334-347

Momany C, Ernst S, Ghosh R, Chang NL, Hackert ML (1995) Crystallographic structure of a PLP-dependent ornithine decarboxylase from Lactobacillus 30a to 3.0 A resolution. J Mol Biol 252: 643-655

Moreau PL (2007) The lysine decarboxylase CadA protects Escherichia coli starved of phosphate against fermentation acids. J Bacteriol 189: 2249-2261

Murshudov GN, Vagin AA, Dodson EJ (1997) Refinement of macromolecular structures by the maximum-likelihood method. Acta Crystallogr D Biol Crystallogr 53 (Pt 3): 240-255

Murzin AG, Brenner SE, Hubbard T, Chothia C (1995) SCOP: a structural classification of proteins database for the investigation of sequences and structures. $J$ Mol Biol 247: 536-540

Neidhardt FC, Bloch PL, Smith DF (1974) Culture medium for enterobacteria. $J$ Bacteriol 119: 736-747

Paiardini A, Bossa F, Pascarella S (2004) Evolutionarily conserved regions and hydrophobic contacts at the superfamily level: the case of the fold-type I, pyridoxal-5'-phosphate-dependent enzymes. Protein Sci 13: 2992-3005 
Park YK, Bearson B, Bang SH, Bang IS, Foster JW (1996) Internal pH crisis, lysine decarboxylase and the acid tolerance response of Salmonella typhimurium. Mol Microbiol 20: 605-611

Potrykus K, Cashel M (2008) (p)ppGpp: still magical? Annu Rev Microbiol 62: 35-51

Richard H, Foster JW (2004) Escherichia coli glutamate- and arginine-dependent acid resistance systems increase internal $\mathrm{pH}$ and reverse transmembrane potential. J Bacteriol 186: 6032-6041

Sabo DL, Boeker EA, Byers B, Waron H, Fischer EH (1974) Purification and physical properties of inducible Escherichia coli lysine decarboxylase. Biochemistry 13: 662-670

Snider J, Gutsche I, Lin M, Baby S, Cox B, Butland G, Greenblatt J, Emili A, Houry WA (2006) Formation of a distinctive complex between the inducible bacterial lysine decarboxylase and a novel AAA + ATPase. J Biol Chem 281: 1532-1546

Soksawatmaekhin W, Kuraishi A, Sakata K, Kashiwagi K, Igarashi K (2004) Excretion and uptake of cadaverine by CadB and its physiological functions in Escherichia coli. Mol Microbiol 51: $1401-1412$

Svitil AL, Cashel M, Zyskind JW (1993) Guanosine tetraphosphate inhibits protein synthesis in vivo. A possible protective mechan- ism for starvation stress in Escherichia coli. J Biol Chem 268: 2307-2311

van Heel M, Harauz G, Orlova EV, Schmidt R, Schatz M (1996) A new generation of the IMAGIC image processing system. $J$ Struct Biol 116: $17-24$

Wallace AC, Laskowski RA, Thornton JM (1995) LIGPLOT: a program to generate schematic diagrams of protein-ligand interactions. Protein Eng 8: 127-134

Wang JD, Sanders GM, Grossman AD (2007) Nutritional control of elongation of DNA replication by (p)ppGpp. Cell 128: 865-875

Watson N, Dunyak DS, Rosey EL, Slonczewski JL, Olson ER (1992) Identification of elements involved in transcriptional regulation of the Escherichia coli cad operon by external pH. J Bacteriol 174: 530-540

Wilks JC, Slonczewski JL (2007) pH of the cytoplasm and periplasm of Escherichia coli: rapid measurement by green fluorescent protein fluorimetry. J Bacteriol 189: 5601-5607

Xiao H, Kalman M, Ikehara K, Zemel S, Glaser G, Cashel M (1991) Residual guanosine $3^{\prime}, 5^{\prime}$-bispyrophosphate synthetic activity of relA null mutants can be eliminated by spoT null mutations. J Biol Chem 266: 5980-5990 